studies (e.g., 7q35, 14q32.33). For example, the most common variant in the Iafrate et al. study, located at 1p21.1 (AMY1A-AMY2A) and present in $>49 \%$ of the individuals studied (Fig. 1), was not detected by Sebat et al. Again, does this discrepancy reflect a technical difference between the two studies or the very different ethnic mix of the two study groups? We will need to study many more individuals from a range of ancestral backgrounds to arrive at an accurate picture of the frequency of the more common LCVs.

\section{LCVs, duplications and disease}

Analysis of the reference sequence shows that $\sim 5 \%$ of the human genome is duplicated ${ }^{2}$. These segmental duplications, defined as multiple regions sharing at least $1 \mathrm{~kb}$ of $90 \%$ identical sequence, are thought to have had a key role in human genome evolution ${ }^{11}$ and may be responsible, through nonallelic homologous recombination (NAHR), for many chromosome rearrangements leading to disease ${ }^{12}$. Both Iafrate et al. and Sebat et al. report a higher than expected association of LCVs with known segmental duplications and with regions associated with human genetic disease or cancer. This suggests that LCVs and other genomic rearrangements might have a common mechanistic basis.

It also has been suggested that large segmental duplications could complicate sequence assembly and lead to gaps in the sequence ${ }^{13}$.
Iafrate et al. note that $12.7 \%$ of LCVs are located in the $100 \mathrm{~kb}$ of gaps in the current sequence assembly. Similarly, LCVs with high sequence homology might be assembled out of the reference sequence. Furthermore, the reference sequence was produced from clone libraries generated from a small number of individuals, and so most LCVs would not be represented in the libraries. This raises the question, "What is the sequence of the normal human genome?" Much more detailed sequence analysis of LCVs in a large number of individuals will be needed to address this issue.

In both studies, a high proportion of LCVs overlapped with known genes. Further studies of these genes in individuals with different copy numbers will be interesting, as copynumber differences will probably be found to influence gene expression ${ }^{14}$. Alternatively, regulatory mechanisms could compensate for differences in copy number between individuals. While LCVs have been identified in phenotypically normal individuals, we cannot determine the phenotypic consequences of such large polymorphisms. Some of these LCVs may be associated with age-related susceptibilities to disease, and deletion polymorphisms may reveal recessive mutations with phenotypic consequences. A recent study of 50 individuals with learning disability and dysmorphology identified five LCVs that were inherited from normal parents and so did not segregate with the disease phenotype ${ }^{15}$. These observations underscore the importance of identifying LCVs in the normal population so that we can gauge the importance of copynumber changes in individuals with diseases.

Both Iafrate et al. and Sebat et al. report that LCVs are frequently located in regions of the genome that are susceptible to rearrangement, particularly by NAHR. The copy-number variation found in LCVs could certainly be generated by NAHR, suggesting that there may be a common mechanism for disease-associated and normal copy-number variation. If this turns out to be true, the LCVs themselves may point to unstable regions of the genome at which new disease-associated rearrangements may be found in the future.

1. Eichler, E.E. \& Frazer, K.A. Genome Biol. 5, 318 (2004).

2. Bailey, J.A. et al. Science 297, 1003-1007 (2002).

3. Campbell, R.D. et al. Exp. Clin. Immunogenet. 7 69-84 (1990)

4. Riley, B. et al. Genomics 79, 197-209 (2002).

5. Blunt, T. et al. Ann. Hum. Genet. 58, 19-24 (1994).

6. Gilles, F. et al. Genomics 70, 364-374 (2000).

7. Hollox, E.J. et al. Am. J. Hum. Genet. 73, 591-600 (2003).

8. Kulski, J.K. et al. Immunol. Rev. 190, 95-122 (2002).

9. lafrate, A.J. et al. Nat. Genet. 36, 949-951 (2004).

10. Sebat, J. et al. Science 305, 525-528 (2004).

11. Bailey, J.A. et al. Genome Biol. 5, R23 (2004).

12. Shaw, C.J. \& Lupski, J.R. Hum. Mol. Genet. $13 \mathrm{Spec}$ No 1, R57-R64 (2004).

13. Eichler, E.E. et al. Nat. Rev. Genet. 5, 345-354 (2004).

14. Townson, J.R. et al. Eur. J. Immunol. 32, 3016-3026 (2002).

15. Shaw-Smith, C. et al. J. Med. Genet. 41, 241-248 (2004).

\title{
Cancer chromosomes in crisis
}

\section{Ronald A. DePinho \& Kornelia Polyak}

The benign-to-malignant transition in human breast cancer is associated with a marked increase in chromosomal aberrations. A new study suggests that telomere dysfunction and its associated bridge-fusion-breakage cycles may drive this episodic instability, thereby providing aspiring cancer cells with the multiple genetic aberrations needed to achieve a fully malignant state.

\author{
Ronald A. DePinho is at the Center for Applied \\ Cancer Science and in the Department of \\ Medical Oncology, Dana-Farber Cancer \\ Institute, Boston, Massachusetts 02115, USA, \\ and the Departments of Medicine and Genetics, \\ Harvard Medical School, Boston, Massachusetts \\ 02115, USA. Kornelia Polyak is in the \\ Department of Medical Oncology, Dana-Farber \\ Cancer Institute, Boston, Massachusetts 02115, \\ USA, and the Departments of Medicine and \\ Genetics, Harvard Medical School, Boston, \\ Massachusetts 02115, USA. \\ e-mail: ron_depinho@dfci.harvard.edu
}

Chromosomal aberrations such as amplifications, deletions and complex translocations are pervasive in human epithelial cancers. These are the main cancers affecting the aged, which has motivated efforts to elucidate the mechanisms leading to chromosomal instability ${ }^{1}$. Several mechanisms of genetic instability have been proposed, including mutations in mitotic checkpoint genes that control chromosome segregation, and loss of telomere capping function resulting in dysfunctional telomeres ${ }^{2}$. The latter model is supported by mouse knock- out studies that have established a link between telomere dysfunction, increased epithelial cancers and radically altered cytogenetic profiles typical of those found in human epithelial cancers ${ }^{3}$. Studies of human primary tumors and epithelial cultures have also supported the idea that telomere dysfunction and its associated bridge-fusion-breakage (BFB) cycles are important in shaping the cancer genome ${ }^{4-6}$. But it is not yet known at which stage of tumorigenesis telomere-induced chromosomal instability unfolds. 


\section{Burst of instability}

On page 984 of this issue, Chin et al. ${ }^{7}$ report that telomere-based BFB events coincide with a burst of chromosomal instability associated with the transition from benign to malignant growth in human breast cancers (Fig. 1). Human breast cancer evolves through a welldefined series of histological stages from normal luminal epithelium to usual ductal hyperplasia (UDH), atypical ductal hyperplasia $(\mathrm{ADH})$, ductal carcinoma in situ (DCIS) and, ultimately, invasive and metastatic cancer. Using fluorescence in situ hybridization, Joe Gray and colleagues observed genome integrity during these early stages of breast cancer development. They identified 'episodic instability' during the transition from ductal hyperplasia to DCIS and, notably, only a limited increase in chromosomal alterations in more advanced disease tissues (invasive cancers). The documentation of telomere erosion and anaphase bridging at this transition, coupled with the known activation of telomerase activity in DCIS and invasive cancers, bolsters the idea that telomere-based crisis is a crucial event in breast tumorigenesis that drives genomic instability. Although only a limited number of tissue samples were analyzed, the data are convincing and reinforced by recent studies describing substantial differences between normal mammary epithelial cells and DCIS, but not between DCIS and invasive tumors ${ }^{8,9}$.

To obtain additional proof that telomerebased crisis fuels genomic instability in breast cancer, Chin et al. analyzed at different passages human mammary epithelial cells immortalized with $Z N F 217$, a putative oncogene on 20q13. As expected, early-passage human mammary epithelial cells were devoid of genetic changes and had normal telomere lengths. But telomeres progressively shortened with passaging and reached a critical length, leading to crisis and telomerase reactivation, around passage 22 . Coincidentally, at this same passage, the frequency of cells with anaphase bridging increased significantly, and numerous genetic changes, similar to the ones detected in primary tumors, became detectable by comparative genomic hybridization, a platform designed to audit regional amplifications and deletions in cancer genomes. Although the cells might have acquired mutations in other genes leading to this change in phenotype, the results suggest that telomere function and genomic instability are linked.

Consistent with the results of Chin et al., previous studies analyzing UDH, DCIS and invasive ductal carcinomas using comparative genomic hybridization detected extensive

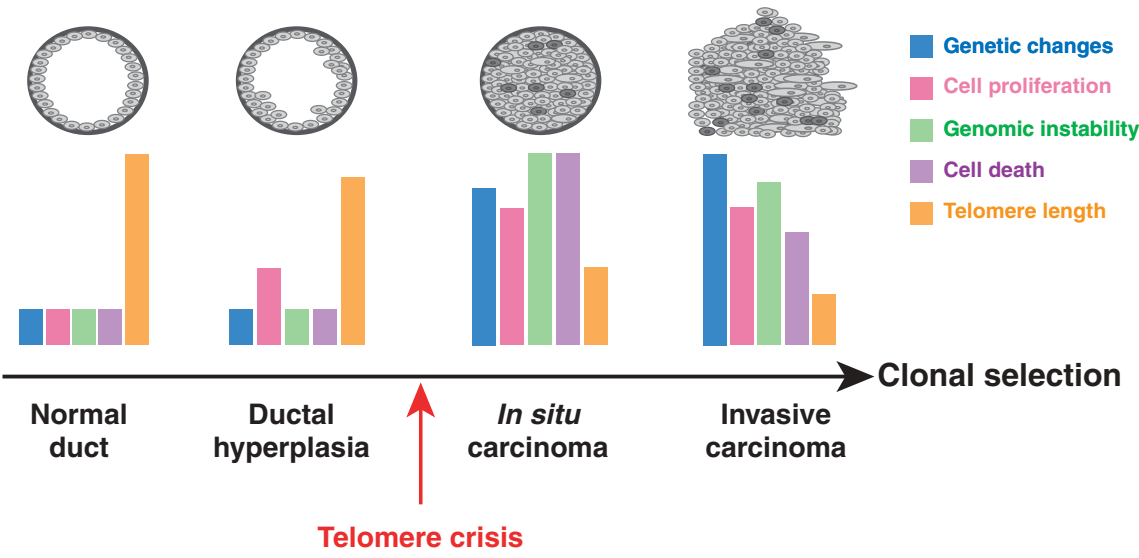

Figure 1 Schematic model of breast tumor progression. Clonal selection drives the accumulation of genetic changes that lead to the development of progressively more aggressive tumor phenotypes. The most notable transition is from ductal hyperplasia (UDH or ADH) to in situ carcinoma (DCIS), with a marked increase in genomic instability, genetic changes and cell death due to shortened telomere-induced crisis (adapted from ref. 7).

recurrent chromosomal changes in both DCIS and invasive lesions, but no such changes in $\mathrm{UDH}^{10}$. Based on these results, the authors of these previous reports speculated that UDH and DCIS are not clonally related, but rather UDH and similar benign tumors might represent a pathologic 'dead end' of tumor evolution. This view is at odds with the model proposed by Chin et al. Although epidemiologic data from individuals with breast cancer support the hypothesis that UDH is not the direct precursor of DCIS, definitive resolution of this point will require further molecular studies in human tumors and model systems.

\section{Paths to aneuploidy}

The model of telomere-linked genomic instability is supported by data from both human tumors and animal models. But crisis is not the sole force driving aneuploidy in epithelial cancers $^{2}$. For example, genes known to have a role in aneuploidy include mitotic spindle checkpoint genes (encoding Bub1, BubR1 and MAD2) and genes involved in recombination and repair (encoding MRE11) and cell cycle control (encoding CDC4 and cyclin E). As each of these genes is mutated in only a small fraction of cases, it seems unlikely that mutations in these genes could provide a rational and unifying explanation for the ageassociated increase in epithelial cancers. In this regard, the role of telomere erosion in driving the age-associated increase in breast cancer incidence becomes important, given the correlation between the number of lifetime menstrual cycles (i.e., amount of epithelial turnover and, hence, telomere attrition) and breast cancer risk.
Even though chromosomal instability and aneuploidy are common features of most cancer types, it is unclear whether genomic instability must be continuously present as tumors progress or whether, after a transient period of crisis resulting in aberrant karyotypes, the cancer genome remains stable unless cells are placed under selective pressure. This latter hypothesis is supported by the finding that DCIS tumors and their invasive local recurrences, or primary tumors and their metachronous metastases, are molecularly similar. Transitory erosion of the telomeres due to sudden clonal expansion may explain the varying degree of genomic instability during tumor progression. At the time of crisis, unprotected chromosome ends can engage in illegitimate recombination, resulting in end-to-end fusion cycles and unstable dicentric chromosomes. In premalignant cells, this would initiate a checkpoint control and lead to elimination by apoptosis. But rare cells could emerge from crisis through activation of telomere maintenance mechanisms or mutations in genes encoding proteins involved in checkpoint control or apoptosis (such as p53; ref. 11). Correspondingly, during breast tumor progression, a sharp increase in apoptosis is observed in DCIS tumors, which declines as they progress to invasive lesions. Thus, telomere dysfunction may have multiple roles in tumorigenesis, contributing in several different ways to the development of the increasingly aggressive tumor phenotype.

The importance of understanding the molecular mechanism of genomic instability goes far beyond solving a crucial and inter- 
esting problem in tumor biology, as instability is one of the main reasons for current therapeutic failures and acquired resistance in cancer therapy. Thus, molecular targeting of pathways responsible for genomic instability or selective killing of cells carrying chromosomal imbalances would have a tremendous impact in the clinical management of individuals with cancer.

1. DePinho, R.A. Nature 408, 248-254 (2000).

2. Storchova, Z. \& Pellman, D. Nat. Rev. Mol. Cell. Biol. 5, 45-54 (2004).

3. Artandi, S.E. et al. Nature 406, 641-645 (2000).

4. Romanov, S.R. et al. Nature 409, 633-637 (2001).

5. Rudolph, K.L. et al. Nat. Genet. 28, 155-159
(2001)

6. van Heek, N.T. et al. Am. J. Pathol. 161, 1541-1547 (2002).

7. Chin, K. et al. Nat. Genet. 36, 984-988 (2004)

8. Ma, X.J. et al. Proc. Natl. Acad. Sci. USA 100, 5974-5979 (2003).

9. Porter, D. et al. Mol. Cancer Res. 1, 362-375 (2003).

10. Boecker, W. et al. J. Pathol. 195, 415-421 (2001).

11. Chin, L. et al. Cell 97, 527-538 (1999).

\title{
TIMP3 checks inflammation
}

\author{
Roy A Black
}

Mice deficient in the metalloprotease inhibitor TIMP3, which inhibits the tumor-necrosis factor alpha (TNF- $\alpha$ )converting enzyme (TACE, also called ADAM17), have elevated levels of TNF and severe inflammation in the liver. This result confirms the physiological importance of the soluble form of TNF and identifies TIMP3 as a crucial regulator of this inflammatory cytokine.

TIMP3 was first identified, inauspiciously, as a chicken protein similar to two wellstudied mammalian inhibitors of matrixdegrading metalloproteases ${ }^{1}$, but its biological importance has become increasingly apparent. It induces apoptosis ${ }^{2}$, inhibits angiogenesis ${ }^{3}$ and impedes cell migration $^{4}$, at least in tissue culture or when overexpressed in vivo. Whether these effects occur physiologically is not known. In a new study on page 969, Fazilat Mohammed and colleagues ${ }^{5}$ show that TIMP3 is a physiological regulator of inflammation. The authors found that mice lacking TIMP3 develop inflamed livers, and that the cause of this inflammation is an increase in TNF- $\alpha$ activity.

All four known TIMPs ${ }^{6,7}$ control the matrix-degrading metalloproteases, which have roles in many normal and pathological processes involving extracellular matrix turnover and cell migration ${ }^{8}$. TIMP3, uniquely, also inhibits TACE, a protease that generates soluble TNF from the cellsurface form of the cytokine $\mathrm{e}^{9-11}$. Consistent with this in vitro activity, TIMP3 blocks the release of TNF from cells in tissue culture ${ }^{12}$. TNF is a key inflammatory mediator that causes severe liver damage by a variety of mechanisms ${ }^{13}$ (Fig. 1). The demonstration by Mohammed et al. ${ }^{5}$ that TIMP3 controls TNF levels in vivo, and that this check prevents spontaneous inflammation, is a landmark in the TIMP, TNF and inflammation fields.

Roy A. Black is at Amgen, 1201 Amgen Court W., Seattle, Washington, USA.

e-mail:blackra@amgen.com

\section{The TIMP3 cascade}

Mohammed et al. readily detected TNF in liver homogenates from Timp $3^{-1-}$ mice, but not in those from wild-type mice or in any other tissues from Timp $3^{-/-}$mice that they tested. TACE activity was greater in liver homogenates from Timp $3^{-1-}$ mice than in those from wildtype mice. They observed predicted consequences of TNF signaling, such as NFKB activation and IL- 6 production, in livers of Timp $3^{-/-}$mice. By 22 months of age, livers of these mice showed lymphocytic infiltrates and necrosis. Timp $3^{-1-}$ mice that also lacked the type I TNF receptor (the primary transducer of the inflammatory effects of TNF) showed none of these signs of inflammation. This finding confirmed that the inflammatory damage was due to TNF activity. Livers of Timp $3^{-1-}$ mice that had undergone a partial hepatectomy had even higher levels of TNF, which persisted well beyond the transient rise seen in livers of wildtype mice that had undergone a partial hepatectomy. Livers of Timp $3^{-1-}$ mice showed severe necrosis after initially normal regeneration,

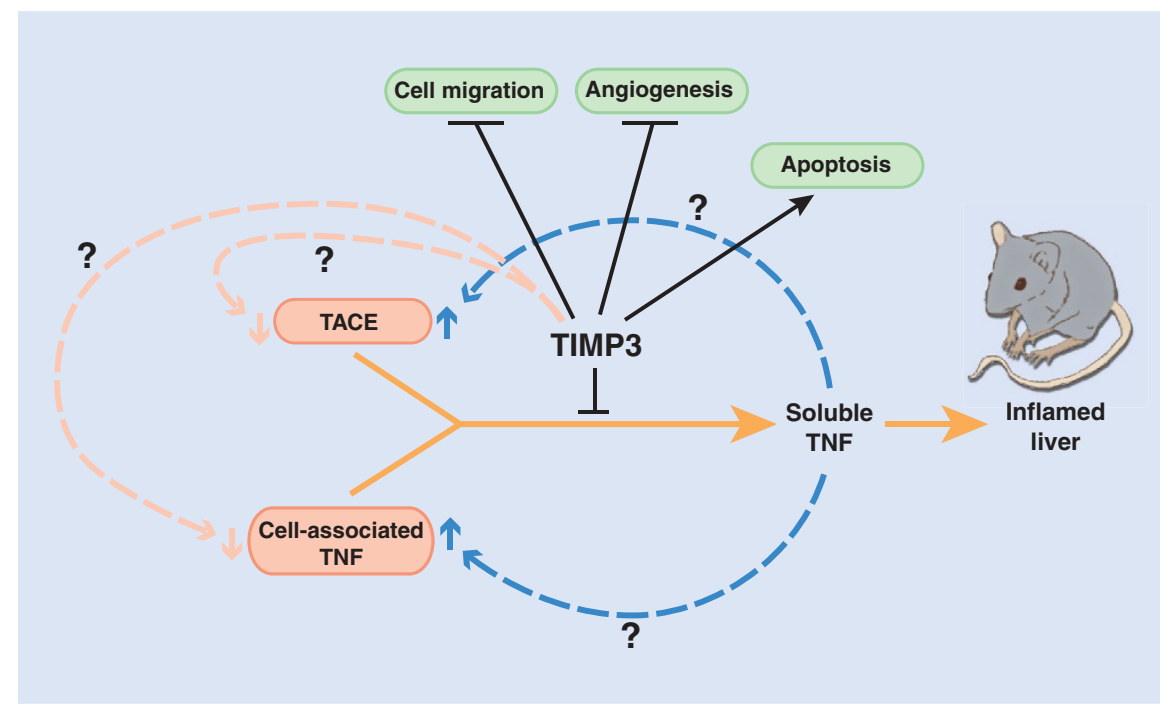

Figure 1 The simplest explanation for the results reported by Mohammed et al. ${ }^{5}$ is that TIMP3 normally inhibits the generation of soluble TNF by TACE, and that the increase in soluble TNF found in Timp $3^{-1-}$ mice leads to inflammation of the liver. The observed increases in the amounts of mature TACE and cell-associated TNF, as well as processed TNF, require further explanation. Soluble TNF might increase the expression of these proteins, either directly or as a result of the inflammation it induces (blue lines). Alternatively, TIMP3 might normally inhibit their expression (pink lines). The effects of TIMP3 on cell migration, angiogenesis and apoptosis (green bubbles) have been observed in tissue culture and upon overexpression in vivo. 